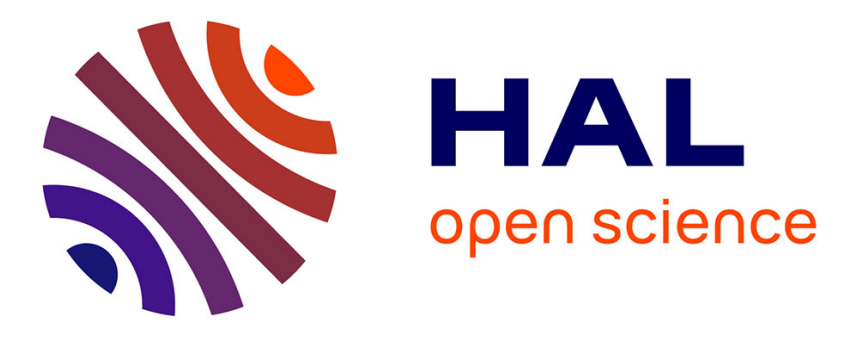

\title{
Two-site double-core-hole states formed when fast protons capture electrons from aligned N2
}

M Gudmundsson, D Fischer, D Misra, A Källberg, A Simonsson, K Støchkel, 4 H Cederquist, $\mathrm{H}$ T Schmidt

\section{- To cite this version:}

M Gudmundsson, D Fischer, D Misra, A Källberg, A Simonsson, et al.. Two-site double-core-hole states formed when fast protons capture electrons from aligned N2. Journal of Physics B: Atomic, Molecular and Optical Physics, 2011, 44 (17), pp.175201. 10.1088/0953-4075/44/17/175201 . hal00651652

\section{HAL Id: hal-00651652 \\ https://hal.science/hal-00651652}

Submitted on 14 Dec 2011

HAL is a multi-disciplinary open access archive for the deposit and dissemination of scientific research documents, whether they are published or not. The documents may come from teaching and research institutions in France or abroad, or from public or private research centers.
L'archive ouverte pluridisciplinaire HAL, est destinée au dépôt et à la diffusion de documents scientifiques de niveau recherche, publiés ou non, émanant des établissements d'enseignement et de recherche français ou étrangers, des laboratoires publics ou privés. 


\title{
Two-site double-core-hole states formed when fast protons capture electrons from aligned $\mathrm{N}_{2}$
}

\author{
M. Gudmundsson, ${ }^{1}$ D. Fischer, ${ }^{2}$ D. Misra, ${ }^{3}$ A. Källberg, ${ }^{1}$ \\ A. Simonsson, ${ }^{1}$ K. Støchkel,${ }^{4}$ H. Cederquist,${ }^{1}$ and H. T. Schmidt ${ }^{1, *}$ \\ ${ }^{1}$ Department of Physics, Stockholm University, S-10691 Stockholm, Sweden \\ ${ }^{2}$ Max-Planck-Institut für Kernphysik, Saupfercheckweg 1, D-69126, Germany \\ ${ }^{3}$ Tata Institute of Fundamental Research, Mumbai, India \\ ${ }^{4}$ Institute of Physics and Astronomy, University of Aarhus, DK-8000 Aarhus C, Denmark
}

(Dated: June 30, 2011)

\begin{abstract}
We report on an experimental investigation of $1.04 \mathrm{MeV} \mathrm{H}^{+}+\mathrm{N}_{2}$ electron transfer collisions. The fast protons were stored in the electron-cooler ion-storage ring, CRYRING and the molecular nitrogen target was provided with a supersonic gas jet. We report momentum distributions of atomic nitrogen dissociation products $\mathrm{N}^{q+}$ with charge states $q+(q=1,2,3)$ which are detected in coincidence with neutralized projectiles. Further, we investigate the influence of the angle between the direction of the incoming projectile beam and the target molecular axis. The orientation of the latter is determined from the momentum vector of one emitted atomic nitrogen fragment ion. We find significantly higher total yields, dominated by $\mathrm{N}^{+}$, of charged atomic dissociation products when the $\mathrm{N}_{2}$ molecular axis is perpendicular to the incoming $\mathrm{H}^{+}$-beam. The relative contributions from $\mathrm{N}^{2+}$ - and $\mathrm{N}^{3+}$ - fragments, however, are strongest when the $\mathrm{N}_{2}$ axis is aligned-or close to aligned - with the ion beam. This, we suggest, is due to increased probabilities for formation of two-site double-core-hole states.
\end{abstract}

\section{INTRODUCTION}

With the development of the COLTRIMS technique (COLd Target Recoil-Ion-Momentum Spectroscopy) in the 1990s an efficient tool for extraction of detailed information on ion - atom collisions became available and revolutionized this area of research [1, 2]. In the case of pure electron-transfer collisions, in which no electrons are emitted from the collision system, analyses of longitudinal recoil-ion momenta have been used to extract detailed final state information [3, 4], while transverse recoil-ion momenta give information on, e.g., very small projectile scattering angles with high precision. For example, the detailed investigations of the Thomas capture mechanism [5-7], its velocity dependence [8] and related phenomena [9] became possible with this technique $[1,2]$.

While the use of COLTRIMS in photo-ionization studies is widespread for both atomic and molecular targets $[10,11]$, there is so far only a very limited number of ion-molecule collision studies. This is somewhat surprising as the detection of the final-state momentum of a charged fragment gives direct information on the orientation of the molecule at the moment of the collision when the time scales for the collision and subsequent dissociation are short compared to molecular rotation periods. There are several examples of theoretical work on ionmolecule collisions revealing interesting features which cannot be isolated without information on the molecular orientation [12, 13]. Fixing or measuring the orientations of target molecules thus open new dimensions for experimental studies of ion-molecule collisions. Cocke

*Electronic address: schmidt@fysik.su.se and co-workers measured the molecular orientation dependence for 2-16 $\mathrm{MeV} \mathrm{O}^{8+}+\mathrm{H}_{2}$ electron transfer collisions by detecting target protons with a pre-COLTRIMS setup in 1993 [14]. This was the first partly successful attempt to establish effects of two-slit projectile wave interference in fast electron-transfer collisions. Much later, similar effects were investigated in more detail by means of a COLTRIMS apparatus where strong interference effects were observed for electron-transfer reactions in $\mathrm{H}^{+}+\mathrm{H}_{2}[15]$ and $\mathrm{He}^{2+}+\mathrm{H}_{2}[16]$ collisions as variations in the total projectile neutralisation cross sections with molecular orientation. Further the two-slit interference was demonstrated more directly through measurement of markedly different projectile angular scattering distributions for different molecular orientations in the $\mathrm{p}+\mathrm{H}_{2}$ case [17]. Also in much lower-energy ionatom collisions heavy-particle interference in the form of Stückelberg oscillations have been clearly observed for electron-transfer collisions $[18,19]$. Other aspects of the hydrogen molecule acting as an atomic sized double-slit have been elucidated for charged-particle-induced pure ionization processes [20-24], for photoionization [11] and for low-energy molecular ion-atom collisions [25].

In the present work we consider electron transfer in $1.04 \mathrm{MeV} p+\mathrm{N}_{2}$ collisions accompanied by emission of one or more additional target electrons. We record the charge state and momentum of one of the atomic product ions from dissociating $\mathrm{N}_{2}$ ions in coincidence with the detection of fast hydrogen atoms formed when electrons are transferred to the proton projectiles. We find that the cross section depends on the molecular orientation, but in different ways for different charge states of the detected atomic fragment ion. For $\mathrm{N}^{+}$fragments, the intensity is highest when the molecular axis is perpendicular to the incoming projectile beam. For the $\mathrm{N}^{2+}$ and 
$\mathrm{N}^{3+}$ fragments, however, the intensities peak for molecular orientations along the beam which most likely is due to higher probabilities to form core holes at both atomic centers for such orientations and thereby to reach higher charge states. The reason for the higher $\mathrm{N}^{+}$intensity for perpendicular orientations is at the moment not clear but we speculate that it possibly may be related to twocenter interference effects as observed earlier for hydrogen targets [15, 17], but here involving inner shell electrons.

\section{EXPERIMENT}

The experiment was performed in the electron cooler heavy-ion storage ring CRYRING at Stockholm University [26]. The protons were produced in a plasma discharge ion source, extracted by a $20 \mathrm{kV}$ potential difference and transported to a linear radio-frequency quadrupole accelerator, which accelerated the beam to $300 \mathrm{keV}$. At this energy the beam was injected into CRYRING and electron cooled to allow injection of further beam pulses to reach total currents of several $\mu \mathrm{A}$. When sufficient beam was accumulated the electron cooler was switched off and the synchrotron acceleration was performed to increase the energy to $1.04 \mathrm{MeV}$. Then, again electron cooling was applied in order to limit the velocity spread in the ion beam and to increase the storage lifetime by eliminating losses through multiple small-angle scattering on residual-gas molecules. For the present experiment we had typical values of beam current of $2 \mu \mathrm{A}$, beam width at the gas-jet interaction region of $1.5 \mathrm{~mm}$ FWHM, and storage lifetimes of 20 minutes. The molecular target beam was formed in a supersonic expansion where pre-cooled $\mathrm{N}_{2}$ gas at 2 bar and $165 \mathrm{~K}$ expanded through a $30 \mu \mathrm{m}$ diameter nozzle into a vacuum chamber pumped to $10^{-3}$ mbar by a $1000 \mathrm{l} / \mathrm{s}$ Balzer's turbomolecular pump. The container with the nozzle could be adjusted with $\mu \mathrm{m}$ precision relative to the first of a set of four skimmers defining the jet and separating the four differential pumping stages toward the storage ring. Opposite to its entrance to the storage ring vacuum system the gas-jet was collected by a three-stage jet dump. The jet was collimated to a diameter of $1.3 \mathrm{~mm}$, a density of $10^{11} \mathrm{~cm}^{-3}$, and it caused no detectable increase in the CRYRING background pressure of $\approx 10^{-11}$ mbar [27].

The crossing of the narrow proton and molecular nitrogen beams is in the center of a recoil-ion momentum spectrometer. A homogeneous electric field is projecting all charged target collision products toward a $95 \%$ transmission grid after which they enter a field-free region where they travel twice the distance they have travelled in the homogeneous field region for Wiley-McLaren time focusing [28]. Finally the ions are accelerated onto a position-sensitve detector consisting of a double microchannel plate in a chevron configuration and a resistive anode encoder. To collect all nitrogen atomic ions from the molecular dissociations, a high extraction field of $200 \mathrm{~V} / \mathrm{cm}$ was applied. As this detector system has no multihit capability it was necessary to cover one half of its surface to only detect one of the two atomic nitrogen fragment ions. In addition, by letting the foil cover a little more than half the detector, we could very strongly reduce a large contribution to the recoil-ion detector count rate from (mostly) single-ionization events yielding $\mathrm{N}_{2}^{+}$ that would have hit close to the center of the detector due to small recoil-ion momenta (in comparison to those of $\mathrm{N}^{q+}$ dissociation products). A schematic of this part of the experimental setup is shown in figure 1. Neutralized projectiles were recorded by a second position-sensitive detector $3.2 \mathrm{~m}$ downstream of the ion beam/gas jet crossing.

The experimental data were recorded in the following manner. After a proton beam has been electron cooled at $1.04 \mathrm{MeV}$, the data acquisition system is enabled. When a signal is registered at the projectile detector an 'open gate' signal is sent to an 8-channel analog-to-digital converter (ADC), the four corner signals of the resistive anode encoder are amplified by charge-sensitive amplifiers and sent to this ADC and a time-to-digital converter (TDC) is started. If a signal is recorded on the recoilion detector, the four corner signals from that detector are also recorded by the ADC, the gate of which is then closed and the time difference between the hits of the two detectors is recorded by the TDC. Finally the ADC and TDC information is transferred by a VME64 crate to an Alpha station and written to an event-mode data file. If no recoil-ion is recorded within a $25 \mu$ s time range, the $\mathrm{ADC}$ and TDC are reset to be ready for the next projectile detection and no information is saved.

In the off-line analysis the charge state of each detected target ion fragment is determined from the time-of-flight. Further, the momentum vector of each fragment ion is calculated from its position on the recoil-ion detectordetermined from the pulse heights of the corner signalsand the time-of-flight.

\section{RESULTS AND DISCUSSION}

In figure 2 we display the histogram of the recorded time differences between signals from the projectile and recoil-ion detectors on a logarithmic scale. We observe atomic fragment ions from dissociations of $\mathrm{N}_{2}$ ions in charge states $q=1-3+$ and there is a narrow peak corresponding to intact molecular nitrogen ions, $\mathrm{N}_{2}^{+}$. This peak corresponds to single-electron capture without further ionization and fragmentation, and is thus likely related to capture of a valence electron from $\mathrm{N}_{2}$. Note that this peak is strongly reduced due to the foil (see figure 1). Even though the recoil-ions in single-electron capture are emitted in the backward direction, their momenta are very small in comparison to those of the atomic fragment ions for which the spectrometer voltages are set, and the vast majority of the $\mathrm{N}_{2}^{+}$ions travel too close to the spectrometer axis to avoid the foil. The considerable broadenings of the atomic $\mathrm{N}^{q+}$ peaks are due to the momentum 


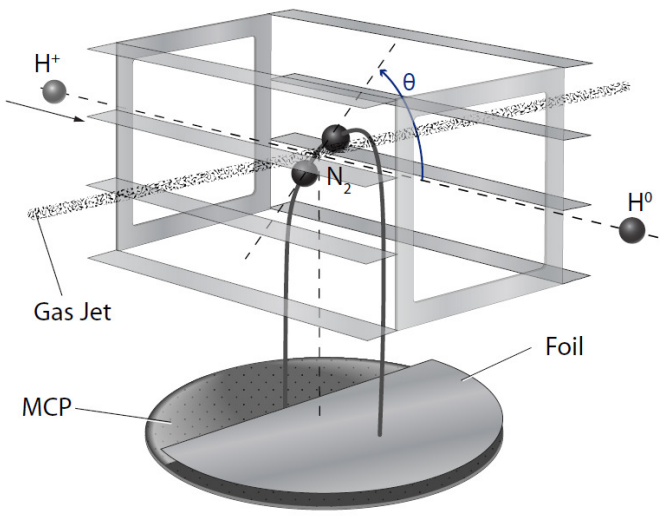

FIG. 1: Schematic of the recoil-ion-momentum spectrometer. The metal foil that covers slightly more than half the detector ensures that only the fragment ion emitted into the backward hemisphere relative to the projectile direction may be detected. This is necessary in order to suppress an otherwise very large backgound from single-ionization events producing mainly intact $\mathrm{N}_{2}^{+}$with low momenta (c.f. text).

gained in the dissociation process itself and it is through the time difference for the individual events in relation to the peak centers that the momentum components along the spectrometer axis are extracted.

It is remarkable that we observe atomic ions up to charge state $q=3+$. Since there is time for the electrons to redistribute themselves during the dissociation, the charge is likely to be shared equally between the two fragments. This equal sharing of the total charge is expected to be a general feature for covalently bound systems and in the case of $\mathrm{N}_{2}$ such an effect has been demonstrated directly in multi-electron transfer processes [29]. Recently it was shown in a free-electron laser experiment that also after sequential multiionization by XUV photons $\mathrm{N}_{2}$ dissociates with symmetrical (or close to symmetrical) sharing of the total charge [30]. Even in some [31], but not all [32] van der Waals bound dimers, it is found that the charge is shared equally. Thus, the present observation of triply charged atomic ions from a covalently bound molecule strongly suggests that at least five electrons were removed from the target molecule in the corresponding collision events. A key to understanding this lies in the fact that the present projectile velocity (6.45 a.u.) is considerably higher than the expectation values of the magnitude of the velocity for the bound $v a$ lence electrons, reducing dramatically the cross section for capture of those. An $\mathrm{N}_{2}$ 1s core electron, on the other hand, is bound by about $400 \mathrm{eV}$, which is then also the expectation value of its kinetic energy in that state. Thus the projectile velocity for $1.04 \mathrm{MeV}$ protons is close to typical speeds of the K-shell electrons of $\mathrm{N}_{2}$ and capture of core electrons is much more likely than

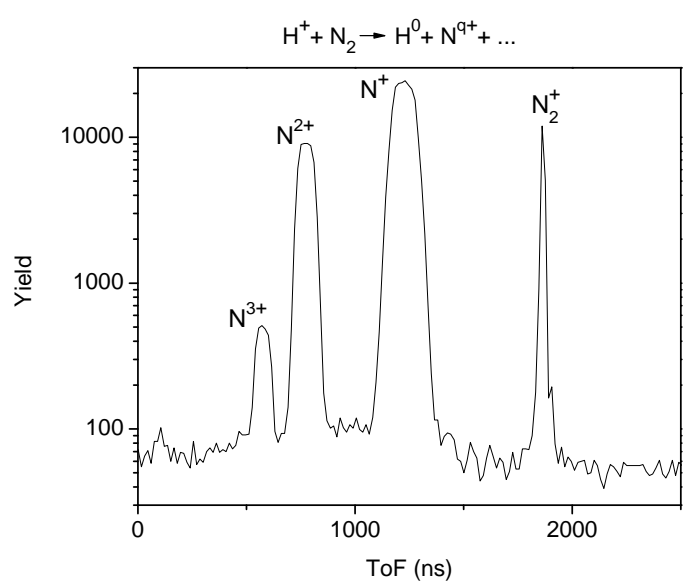

FIG. 2: Time-of-flight spectrum of target ions and fragments recorded in coincidence with neutralized projectiles. Note that the $\mathrm{N}_{2}^{+}$peak corresponding to molecular ions is strongly reduced due to the coverage of a little more than one half of the detector area by a foil (cf. text).

capture of valence electrons. Core-hole states will decay through Auger processes and the multiply charged molecular ion will then rapidly dissociate after redistribution of the charge. For photoionization above the threshold for $\mathrm{K}$-shell ionization, a similar three-step mechanism (Khole formation-Auger decay-Coulomb dissociation) for the formation of multiply charged atomic fragments has been discussed [33]. This emphasizes interesting similarities between electron transfer in fast collisions with ions and photoionization as discussed also in [34].

The distributions of the magnitudes of final-state momenta of the atomic nitrogen fragment ions are shown in figure 3 for the $\mathrm{N}^{+}, \mathrm{N}^{2+}$ and $\mathrm{N}^{3+}$ dissociation products. As expected, the more highly charged ions also have higher momenta due to the Coulomb repulsion. As we only detect one of the atomic fragment ions we do not know the total number of electrons removed in each event, but we do observe for example that the maximum in the $\mathrm{N}^{2+}$ distribution coincides with a 'shoulder structure' in the $\mathrm{N}^{+}$momentum distribution. This indicates that this $\mathrm{N}^{+}$-structure is related to dissociating $\mathrm{N}_{2}^{3+}$. The structures in the $\mathrm{N}^{+}$distribution for lower momenta are probably due to different dissociating states of $\mathrm{N}_{2}^{+}$and $\mathrm{N}_{2}^{2+}$. The high-momentum tails of the $\mathrm{N}^{2+}$ and $\mathrm{N}^{3+}$ distributions seem to coincide and this would indicate that $\mathrm{N}_{2}^{5+} \rightarrow \mathrm{N}^{2+}+\mathrm{N}^{3+}$ is responsible for the highest kinetic energies in both cases. Thus, $\mathrm{N}_{2}^{5+}$ should be the most highly charged molecule produced in this experiment, and given the equal sharing of charge we may conclude that the observed $\mathrm{N}^{3+}$ ions come from dissociating $\mathrm{N}_{2}^{5+}$ exclusively.

In figure 4 we show the orientation dependence in $\mathrm{d} \sigma / \mathrm{d}(\cos \theta)$ summed over all three observed atomic charge states, where $\theta$ is the angle between the direc- 


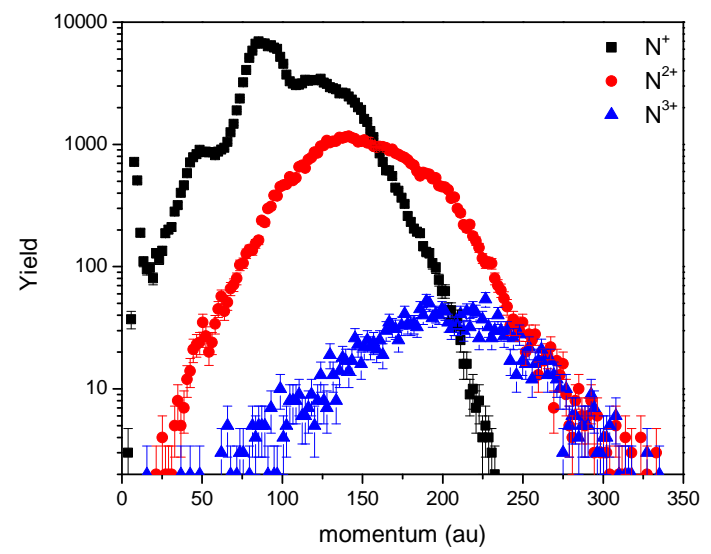

FIG. 3: The distributions of the absolute values of the momenta for the three different charge states of atomic fragments from dissociations of $\mathrm{N}_{2}$ ions.

tion of emission of the detected dissociation product and the projectile beam direction. The sharp cut in the signal for $\cos (\theta) \geq-0.1$ is due to the foil that covers a little over one half the detector area. We find a significant variation with the angle $\theta$ with about $50 \%$ higher signal for the molecular axis close to perpendicular to the beam direction as compared to the molecular axis aligned with the beam direction. In earlier studies of transfer and excitation in $\mathrm{p}+\mathrm{H}_{2}$ collisions [15] a similar strong orientation dependence could be explained by the welldefined changes in projectile momentum (and thus deBroglie wavelength) that accompanies the electron capture in the vicinity of either target proton. For capture of a target electron from an initial state of gerade symmetry it was predicted $[35,36]$ and later found $[15,17]$ that constructive interference gives a maximum in the cross section for the transverse orientation of the molecular axis with respect to the incoming projectile direction. The same reasoning predicts that there should be minima due to destructive interferences at other specific orientations $(\theta)$ determined by the momentum gained by the projectile $\left(\approx v_{P} / 2\right.$, where $v_{P}$ is the projectile velocity) when an electron is captured from $\mathrm{H}_{2}$. Such projectileenergy dependent minima were clearly identified in [15]. It was further shown [37] that the inverse orientation dependence should be expected when an electron from an intial state of ungerade symmetry is captured. The hydrogen molecule, $\mathrm{H}_{2}$, is unique in that both electrons (neglecting small configuration interaction effects) occupy single-electron states of gerade symmetry. In a larger system like $\mathrm{N}_{2}$ single-electron states of both symmetries are occupied and similar interference as observed in electron capture from $\mathrm{H}_{2}$ can only be expected in the case of a strong preference for capture from states of either initial symmetry.

The longitudinal momentum transfer to the projec-

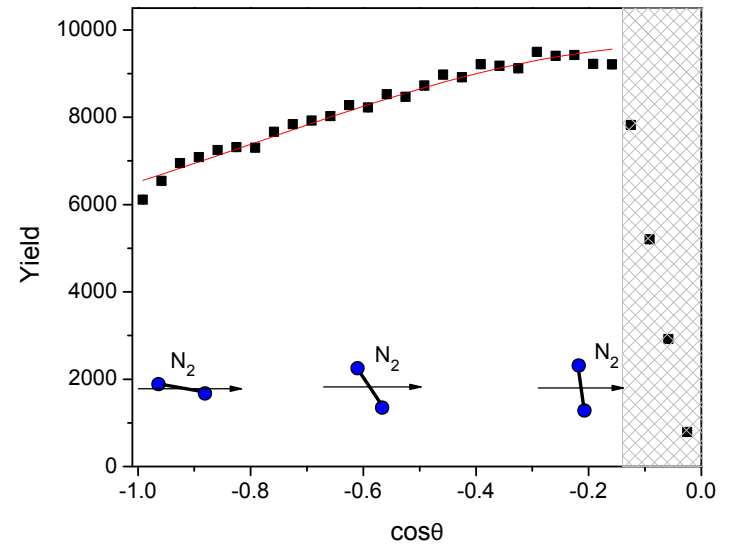

FIG. 4: The measured orientation-dependent relative cross section $d \sigma / d \cos \theta$ as functions of $\cos \theta$ summed over all three observed atomic ion charge states. This entity would be constant for an isotropic distribution of the emitted atomic nitrogen ions.

tile in the initial electron-transfer process is given by (in atomic units):

$$
\delta k=v_{\mathrm{P}} / 2+Q / v_{\mathrm{P}},
$$

where $Q$ is the inelasticity of the collision. In atomic units the change in momentum is equal to the change in wave number of the de Broglie wave and the phase shift accumulated as the wave front propagates from one nuclear center to the other is given by:

$$
\delta \Phi=\delta k a \cos \theta
$$

where $a=2.1 a_{0}$ is the internuclear distance in $\mathrm{N}_{2}$.

In the case of capture of a valence electron, the $Q / v_{P^{-}}$ term in (1) is negligible as was the case for capture from $\mathrm{H}_{2}$. Thus $\delta k a \approx 6.7$ for valence capture from $\mathrm{N}_{2}$ and the phase shift would vary from zero (for capture from a gerade symmetry initial state) to more than $-2 \pi$ when the molecular axis orientation is varied from transverse $\left(\theta=90^{\circ}, \delta \Phi=0\right)$ to parallel $\left(\theta=180^{\circ}, \delta \Phi=-\delta k a\right)$. Thus if capture of initial gerade symmetry valence electrons would dominate, we should find a variation with a maximum at $\cos \theta=0$, an intermediate minimum and another maximum close to the parallel orientation. Such a behaviour is definitely ruled out from the data shown in figure 4.

In the case of inner-shell capture from $\mathrm{N}_{2}$, on the other hand, the $Q / v_{P}$-term is significant and the momentum gained by the projectile is strongly reduced so that $\delta k a \approx 2.0$ according to $(1)$. Therefore, a minimum due to destructive interference $(\delta \Phi=-\pi)$ is never reached when the molecular axis orientation is varied. Rather we go from a maximum at $\theta=90^{\circ}$ towards lower intensity when approaching $\theta=180^{\circ}$. Our observations resembles 


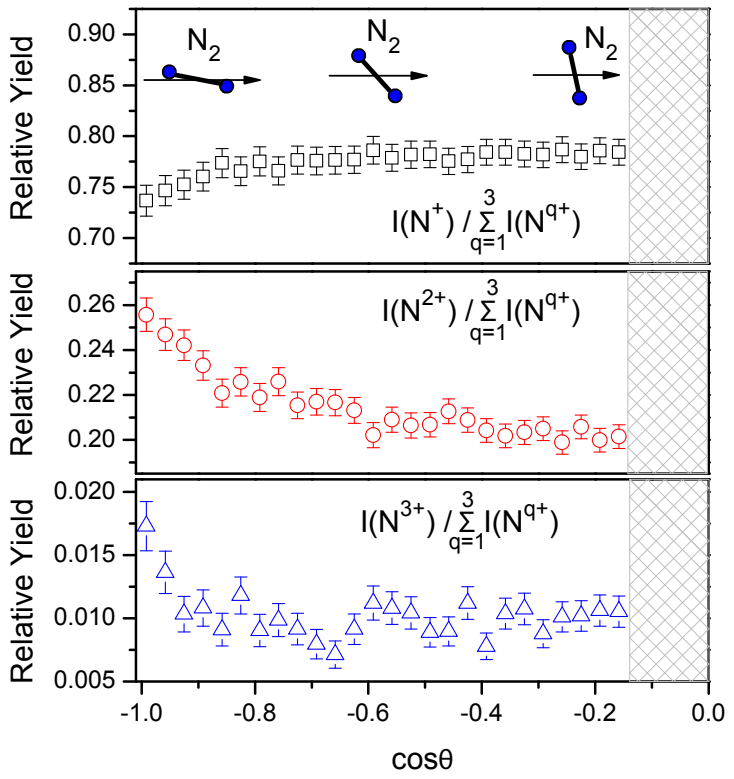

FIG. 5: The relative yields of $\mathrm{N}^{+}, \mathrm{N}^{2+}$ and $\mathrm{N}^{3+}$ as functions of $\cos \theta$.

this behaviour as is shown in figure 4 . This observed behavior is what is expected for capture of electrons from an initial state of gerade symmetry and consistent with what was found experimentally for $\mathrm{p}+\mathrm{H}_{2}[15,17]$. As mentioned above the expectation for capture of electrons from states of initial ungerade symmetry will be the reverse, and thus our experimental result seems to indicate that there is a higher probability of capture of an electron in an initial gerade state. The full curve in figure 4 is the result of treating the projectile wave functions after the electron transfer process as the sum of two plane waves with a phase shift given by (2) for a case where capture of electrons initially occupying states of gerade symmetry is dominating. There is good agreement between the data and this simple model result, but rather than drawing any conclusions from this we propose further experimental investigations to be made. In the $\mathrm{p}+\mathrm{H}_{2}$ case [15], the projectile-wave interference was nicely demonstrated by the variation of the position of the minimum with projectile velocity. In the case of $\mathrm{p}+\mathrm{N}_{2}$ core-electron capture a $2.0 \mathrm{MeV}$ projectile kinetic energy would give $\delta k a \approx 6.0$ and a minimum in the orientation dependent cross section for $\theta \approx 58^{\circ}$ as an example. It would be really interesting if such an experiment could be performed in the near future - unfortunately it cannot be done with the present setup for practical reasons as the CRYRING is no longer available for experiments.

Irrespective of how the orientation variations of $\mathrm{d} \sigma / d \cos \theta$ in figure 4 should be understood, we may consider whether these dependences differ for different atomic ion fragment charge states. For this purpose, the relative yields of $\mathrm{N}^{+}, \mathrm{N}^{2+}$ and $\mathrm{N}^{3+}$ to the summed yields are shown in figure 5 as functions of $\cos \theta$ where $\theta$ again is the angle of emission of the detected fragment. We find that multiply charged atomic fragments are more likely to be found in electron transfer events where $\cos \theta$ is close to -1, i.e. for the molecular axis aligned with the projectile beam. This means that there is a higher probability for higher atomic fragment ion charge states for this orientation and we ascribe this to steric effects. When a projectile comes sufficiently close to one nuclear center for a high probability for electron transfer, it must also come close to the other atomic center for molecules close to parallel with the ion beam, which will increase the probability for impact ionization of a K-shell electron at the other atomic center. In a scenario where one core hole is created through electron transfer and another core hole is created at the other nuclear center by impact ionization, it is natural that high charge states are formed. Currently, such two-site double-core hole states are being sought for in sequential X-ray photoionization by means of the Stanford Linear Coherent Light Source free-electron X-ray laser, while single-site molecular nitrogen double core hole states produced with that technique [38] and in single X-ray photon experiments $[39,40]$ have been reported recently.

\section{CONCLUSION}

We have reported the results of relative cross section measurements differential in the orientation of the molecular axis for electron transfer processes in $1.04 \mathrm{MeV} \mathrm{p} \mathrm{+}$ $\mathrm{N}_{2}$ collisions. We find that electron transfer leading to dissociation into charged products is more likely if the molecular axis is oriented perpendicular to the incoming $\mathrm{H}^{+}$trajectory. This behaviour resembles that found for molecular hydrogen target molecules in earlier work and may possibly be explained by de Broglie wave interference due to electron transfer in close vicinities of either atomic core. If this is indeed the case, it is a very surprising result as such interferences should be unique for molecular hydrogen as only there both electrons are in gerade-symmetry initial single-electron states. Rather than concluding that quantum interference is responsible for the observed orientational variation of the cross sections, we propose to repeat the experiment at a higher projectile beam energy around $2 \mathrm{MeV}$ in order to investigate if there is also a minimum (around $\theta=58^{\circ}$ ) when the molecular orientation is varied between parallel and perpendicular orientations. In the present work we have observed that the relative intensities of the $\mathrm{N}^{2+}$ and $\mathrm{N}^{3+}$ dissociation products increase significantly for orientations close to aligned with the direction of the incoming ion beam. We ascribe this observation to the formation of two-site double-core-hole states formed when one core electron is captured while another one from the other atomic core is ionized by the projectile. 


\section{Acknowledgments}

Financial support from the Knut and Alice Wallenberg Foundation and the Swedish Research Council is gratefully acknowledged. Further, we would like to acknowledge many fruitful discussions with Charles Lewis Cocke, Erik Horsdal, Horst Schmidt-Böcking, and Reinhold Schuch.
[1] R. Dörner, V. Mergel, O. Jagutzki, L. Spielberger, J. Ullrich, R. Moshammer, and H. Schmidt-Böcking, Phys. Rep. 330, 95 (2000).

[2] J. Ullrich, R. Moshammer, A. Dorn, R. Dörner, L. Ph. H. Schmidt, and H. Schmidt-Böcking, Rep. Prog. Phys. 66, 1463 (2003).

[3] V. Mergel, R. Dörner, J. Ullrich, O. Jagutzki, S. Lencinas, S. Nüttges, L. Spielberger, M. Unverzagt, C. L. Cocke, R. E. Olson, et al., Phys. Rev. Lett. 74, 2200 (1995).

[4] A. Cassimi, S. Duponchel, X. Flechard, P. Jardin, P. Sortais, D. Hennecart, and R. E. Olson, Phys. Rev. Lett. 76, 3679 (1996).

[5] L. H. Thomas, Proc. R. Soc. 114, 561 (1927).

[6] E. Horsdal-Pedersen, C. L. Cocke, and M. Stockli, Phys. Rev. Lett. 50, 1910 (1983).

[7] D. Fischer, K. Støchkel, H. Cederquist, H. Zettergren, P. Reinhed, R. Schuch, A. Källberg, A. Simonsson, and H. T. Schmidt, Phys. Rev. A 73, 052713 (2006).

[8] D. Fischer, M. Gudmundsson, Z. Berényi, N. Haag, H. A. B. Johansson, D. Misra, P. Reinhed, A. Källberg, A. Simonsson, K. Støchkel, et al., Phys. Rev. A 81, 012714 (2010).

[9] M. Gudmundsson, D. Fischer, N. Haag, H. A. B. Johansson, D. Misra, P. Reinhed, H. Schmidt-Böcking, R. Schuch, M. Schöffler, K. Støchkel, et al., Journal of Physics B: Atomic, Molecular and Optical Physics 43, 185209 (2010).

[10] H. Schmidt-Böcking, R. Dörner, and J. Ullrich, Europhysics News 33, 210 (2002).

[11] D. Akoury, K. Kreidi, T. Jahnke, T. Weber, A. Staudte, M. Schöffler, N. Neumann, J. Titze, L. P. H. Schmidt, A. Czasch, et al., Science 318, 949 (2007).

[12] T. F. Tuan and E. Gerjuoy, Phys. Rev. 117, 756 (1960).

[13] N. C. Deb, A. Jain, and J. H. McGuire, Phys. Rev. A 38, 3769 (1988).

[14] S. Cheng, C. L. Cocke, V. Frohne, E. Y. Kamber, J. H. McGuire, and Y. Wang, Phys. Rev. A 47, 3923 (1993).

[15] K. Støchkel, O. Eidem, H. Cederquist, H. Zettergren, P. Reinhed, R. Schuch, C. L. Cocke, S. B. Levin, V. N. Ostrovsky, A. Källberg, et al., Phys. Rev. A 72, 050703(R) (2005).

[16] D. Misra, H. T. Schmidt, M. Gudmundsson, D. Fischer, N. Haag, H. A. B. Johansson, A. Källberg, B. Najjari, P. Reinhed, R. Schuch, et al., Phys. Rev. Lett. 102, 153201 (2009)

[17] H. T. Schmidt, D. Fischer, Z. Berenyi, C. L. Cocke, M. Gudmundsson, N. Haag, H. A. B. Johansson, A. Källberg, S. B. Levin, P. Reinhed, et al., Phys. Rev. Lett. 101, 083201 (2008).

[18] A. Bárány, H. Danared, H. Cederquist, P. Hvelplund, H. Knudsen, J. O. K. Pedersen, C. L. Cocke, L. N. Tunnell, W. Waggoner, and J. P. Giese, Journal of Physics B: Atomic and Molecular Physics 19, L427 (1986).

[19] M. Hoshino, L. Pichl, Y. Kanai, Y. Nakai, M. Kitajima,
M. Kimura, Y. Li, H.-P. Liebermann, R. J. Buenker, H. Tanaka, et al., Phys. Rev. A 75, 012716 (2007).

[20] R. O. Barrachina, F. Frémont, K. Fossez, D. Gruyer, V. Helaine, A. Lepailleur, A. Leredde, S. Maclot, G. Scamps, and J.-Y. Chesnel, Phys. Rev. A 81, 060702 (2010).

[21] J.-Y. Chesnel, A. Hajaji, R. O. Barrachina, and F. Frémont, Phys. Rev. Lett. 98, 100403 (2007).

[22] D. Misra, A. Kelkar, U. Kadhane, A. Kumar, L. C. Tribedi, and P. D. Fainstein, Phys. Rev. A 74, 060701 (2006).

[23] N. Stolterfoht, B. Sulik, V. Hoffmann, B. Skogvall, J. Y. Chesnel, J. Rangama, F. Frémont, D. Hennecart, A. Cassimi, X. Husson, et al., Phys. Rev. Lett. 87, 023201 (2001).

[24] N. Stolterfoht, B. Sulik, B. Skogvall, J. Y. Chesnel, F. Frémont, D. Hennecart, A. Cassimi, L. Adoui, S. Hossain, and J. A. Tanis, Phys. Rev. A 69, 012701 (2004).

[25] L. Ph. H. Schmidt, S. Schössler, F. Afaneh, M. Schöffler, K. E. Stiebing, H. Schmidt-Böcking, and R. Dörner, Phys. Rev. Lett. 101, 173202 (2008).

[26] K. Abrahamsson, G. Andler, L. Bagge, E. Beebe, P. Carlé, H. Danared, S. Egnell, K. Ehrnstén, M. Engström, C. J. Herrlander, et al., Nucl. Inst. \& Meth. in Phys. Res. B 79, 269 (1993).

[27] H. T. Schmidt, H. Cederquist, R. Schuch, L. Bagge, A. Källberg, J. Hilke, K.-G. Rensfelt, V. Mergel, M. Achler, R. Dörner, et al., Hyp. Int. 108, 339 (1997).

[28] W. C. Wiley and I. H. McLaren, Review of Scientific Instruments 26, 1150 (1955).

[29] A. Remscheid, B. A. Huber, M. Pykavyj, V. Staemmler, and K. Wiesemann, Journal of Physics B: Atomic, Molecular and Optical Physics 29, 515 (1996).

[30] Y. H. Jiang, T. Pfeifer, A. Rudenko, O. Herrwerth, L. Foucar, M. Kurka, K. U. Kühnel, M. Lezius, M. F. Kling, X. Liu, et al., Phys. Rev. A 82, 041403 (2010).

[31] H. Zettergren, H. T. Schmidt, P. Reinhed, H. Cederquist, J. Jensen, P. Hvelplund, S. Tomita, B. Manil, J. Rangama, and B. A. Huber, Phys. Rev. A 75, 051201 (2007).

[32] J. Matsumoto, A. Leredde, X. Flechard, K. Hayakawa, H. Shiromaru, J. Rangama, C. L. Zhou, S. Guillous, D. Hennecart, T. Muranaka, et al., Phys. Rev. Lett. 105, $263202(2010)$.

[33] T. Weber, O. Jagutzki, M. Hattass, A. Staudte, A. Nauert, L. Schmidt, M. H. Prior, A. L. Landers, A. Bräuning-Demian, H. Bräuning, et al., Journal of Physics B: Atomic, Molecular and Optical Physics 34, 3669 (2001).

[34] H. T. Schmidt, A. Fardi, R. Schuch, S. H. Schwartz, H. Zettergren, H. Cederquist, L. Bagge, H. Danared, A. Källberg, J. Jensen, et al., Phys. Rev. Lett. 89, 163201 (2002).

[35] R. Shingal and C. D. Lin, Phys. Rev. A 40, 1302 (1989).

[36] Y. D. Wang, J. H. McGuire, and R. D. Rivarola, Phys. 
Rev. A 40, 3673 (1989).

[37] O. Eidem

Masters

Thesis,

Stockholm University, http://www.fysik.su.se/ schmidt/Eidem.pdf.

(2005),

38] J. P. Cryan, J. M. Glownia, J. Andreasson, A. Belkacem N. Berrah, C. I. Blaga, C. Bostedt, J. Bozek, C. Buth, L. F. DiMauro, et al., Phys. Rev. Lett. 105, 083004 (2010).
[39] P. Lablanquie, F. Penent, J. Palaudoux, L. Andric, P. Selles, S. Carniato, K. Bučar, M. Žitnik, M. Huttula, J. H. D. Eland, et al., Phys. Rev. Lett. 106, 063003 (2011).

[40] J. H. D. Eland, M. Tashiro, P. Linusson, M. Ehara, K. Ueda, and R. Feifel, Phys. Rev. Lett. 105, 213005 (2010). 